\title{
Research on Machine Vision Detection Experiment Platform for Industry Products
}

\author{
Peng $\mathrm{Ye}^{1}$, Xingyu Gao ${ }^{2, \mathrm{a},{ }^{*}}$ \\ ${ }^{1}$ College of Electronic Engineering and Automation, Guilin University of Electronic Technology, \\ Guilin 541004,China; \\ ${ }^{2}$ College of Electromechanical Engineering, Guilin University of Electronic Technology, Guilin \\ 541004,China. \\ a, ${ }^{\star}$ gxy1981@guet.edu.cn
}

Keywords: Parts inspection,Machine vision detection,Focusing evaluation function

\begin{abstract}
Key technologies of machine vision detection system for the industrial part are investigated and an intact set of high-speed detection system is developed. On the basis of introducing the entire design of machine vision platform, analyzing the software and hardware structure and fundamental principles of control units, this paper proposes that variance function shall be adopted for coarse tuning and the search algorithm of Roberts gradient sum function shall be adopted for fine tuning. The experimental results show that it only takes 3.246 second for this system to collect images, which meets the requirement of real-time online detection.This system is feasible for the detection experiments of industrial products.
\end{abstract}

\section{Introduction}

During the mass production process of industrial parts, in order to ensure the quality of finished parts and improve the productivity, it is necessary to detect $100 \%$ products online[1].However, due to the diversification of industrial parts[2], their detecting requirements are different and the detecting process has become increasingly difficult.The traditional manual detection is not only time- and effort-consuming but also cannot guarantee the accuracy and speed of detection[3].Machine vision detection technology has the advantages of non-contact, high precision, high speed, automation and intelligence[4], and is easy to be integrated with design and process control information, which will satisfy the needs of parts detection.Therefore, developing high-speed and universal machine vision detection system with self-owned intellectual property is of great theoretical significance and of high value of application.

In this paper, we demonstrate a general machine vision detection platform, which consists of a camera with high performance lens, a light source that can change the illumination modes, a conveyer belt that takes the parts to the detection position, and a computer to control all the moving parts and process the digital images. The automatic focusing is realized by the motor that controlled by images blur function. A detection experiment is carried out which testifies the detection ability of this platform.

\section{Schematic design of the general machine vision detection platform}

In this paper, the design of machine vision platform is a common type of multi-function visual inspection laboratory equipment. As shown in figure 1, when tested items moving through the conveyor belt into the detection area, the laser sensor is triggered. When laser sensor receives the signal of the part in position, a feedback signal is sent to the PC. The PC sends an image collection signal, which orders the CCD camera to capture the image of the part. The image is transferred to the memory of the PC. Using image processing algorithm, the image is analyzed by the blur function to judge whether the part locates on the object plane of the lens. If not, the $\mathrm{Z}$ axis motor drives the camera to move up or down to find another plane. The above steps carries out again and again, until the best object plane is found. Then the auto-focusing process is finished. After that, 
the image process algorithm detects the defects of the parts. If no defect found, then this part is qualified. If any defects found, this part is un-qualified which will be selected out from the product line by the pneumatic clamping hand[5]. On the whole system platform, the parameters of the camera and lens, the light source and the sensor are selected according to the detecting requirements of the tested object, which realizes the multi-function general on-line detection.

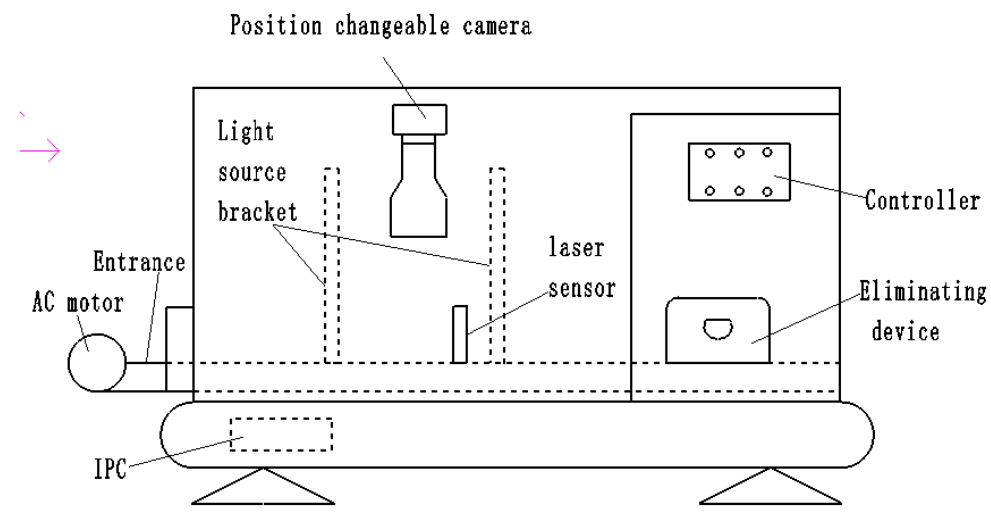

Fig.1 Overallstructure block diagram

\section{The hardware design}

As shown in figure 2, the machine vision detection platform is divided into image acquisition system, control system and screening system. The image acquisition system is composed of an industrial camera, a lens, a light source and the image processing equipment. Industrial camera uses the commercial MV-EM500M camera, with the CCDtarget surface size of 1/2.5 ", a resolution of $2592 \times 1944$, and a pixel size of $2.2 \times 2$.2um. A telecentric lens with a field of view $200 \mathrm{~mm}$ is used. The lens is installed on the perpendicular to the sliding table at the bottom of the module, overlooking the platform and through the step motor to adjust operation. The illumination light source is an adjustable LED strip light, which can provide the oblique and uniform illumination for the objects from any angle. The computer deals with the image data after the corresponding instructions sent to the control system.

The control system mainly includes the lens focus motor, transmission platform, laser sensor, light source and filter system control. The working mode of the control system is based on PC and the micro controller. The PC controls the program editing, user interface management, etc. Micro controller is used to manage the subroutine, and is responsible for motion control of manipulator and logic control, allows users to develop and expand, and has upper and lower two levels of openness.

Center control unit uses the STM32f103RCT6 [6] as the main control chip, which is a 32 bit microprocessor and has great power consumption control with a number of peripherals based on ARM kernel architecture M3. The MCU working frequency is $72 \mathrm{MHz}$, working voltage is 2.0 3.6 V with seven 16-bit timer, has 2 ADC 13 communication interfaces, can Support USART, SPI, CAN, USB and other communication interfaces, also can realize infrared, CAN bus, GPRS and other data transmission, which meets the functional requirements. The chip hardware has single cycle of the multiplier, which greatly improves the operation speed. Its code density saves 30\% $45 \%$ than the ordinary 32-bit MCU, 50\% than 16-bit single chip province. 


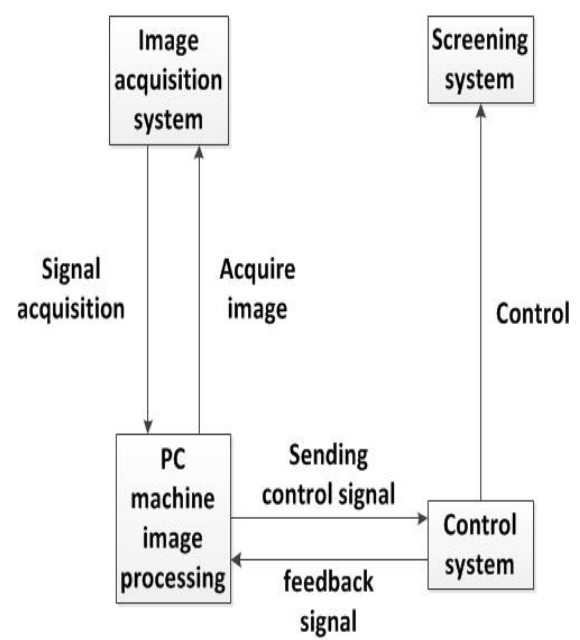

Fig.2 Control system structure

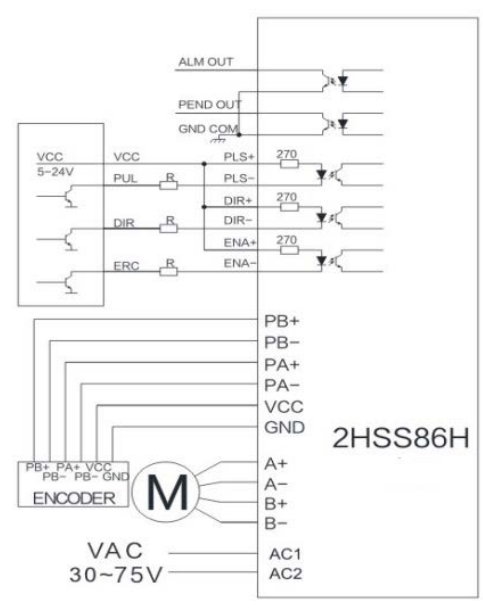

Fig. 3 Stepper motor drives

The lens focusing and screening system uses the 57 series and 84 series of stepper motor [7]. As shown in figure 3 , the system drive is a $2 \mathrm{HSS} 86 \mathrm{H}$ closed-loop stepper motor drive. $2 \mathrm{HSS} 86 \mathrm{H}$ hybrids stepping servo drive system mix the servo control system in digital stepper driver.Product uses the classic three-loop control method (position loop, speed loop and current loop), it compatibles with the characteristics of the step motor and servo motor. The drive has a light isolation fault alarm output interface ALM, with nearly $100 \%$ of the torque output. Segment set range is 2-256, manually dial the code segment for 800-40000.

\section{The software design}

Because the machine vision detection equipment is an online detection system, the test time must keep synchronization with high-speed production line. The key of the synchronization technology is how image acquisition system and screening system effectively running at a high speed. When the tested parts are transferred through the conveyor belt to the image acquisition position, the laser sensor sends a feedback signal to the center control unit, which command the camera to capture an image. Because this system is suitable for a variety kinds of parts, for the first time of detection of each kind of parts, it is necessary to carry on the automatic focusing. So that to make the collected images reach the most clear level, and improves the filtering accuracy.

Recent years, many kinds of focusing function have been put forward[8], but each focusing function only has a good effect for one or several kinds of image. The Variance function is suitable for a wide range of rough focusing because of its larger focusing range.The Roberts gradient sum function is suitable for small range of automatic focus, which has good stability and high sensitivity near the focus location.

The traditional single times full image search algorithm[9] can't satisfy the requirements of this system because of the waste of time. The auto-focusing algorithm for the combination of coarse tuning and fine-tuning are designed based on the characteristics of Roberts gradient function and Variance function. First, the step motor runs from the initial position with a large step, the camera record the images of each step. The Variance function calculates the current definition $f n$. The definition of the last step is compared with that of the previous step. This process continues until 2 times less happened. At this time, the maximum resolution is found out, and the camera quickly transferred to the maximum corresponding position, which means the coarse adjustment is completed.

The stepper motor runs forward with smaller step from the current position. The current definition $\mathrm{g} 1$ is calculated by the Roberts gradient sum function definition. If sharpness value increases after the definition of value compared with the previous definition of the collection value, motor should continue running to be the same direction. If definition value decreases, the motor should run back. At this point motor sets the step length to 0.5 times of the last step. When motor finds the peak, the step decrease to half. This process continues until the definition within the 
threshold value, then the auto-focusing completes. The program flow chat is shown in Fig.4.
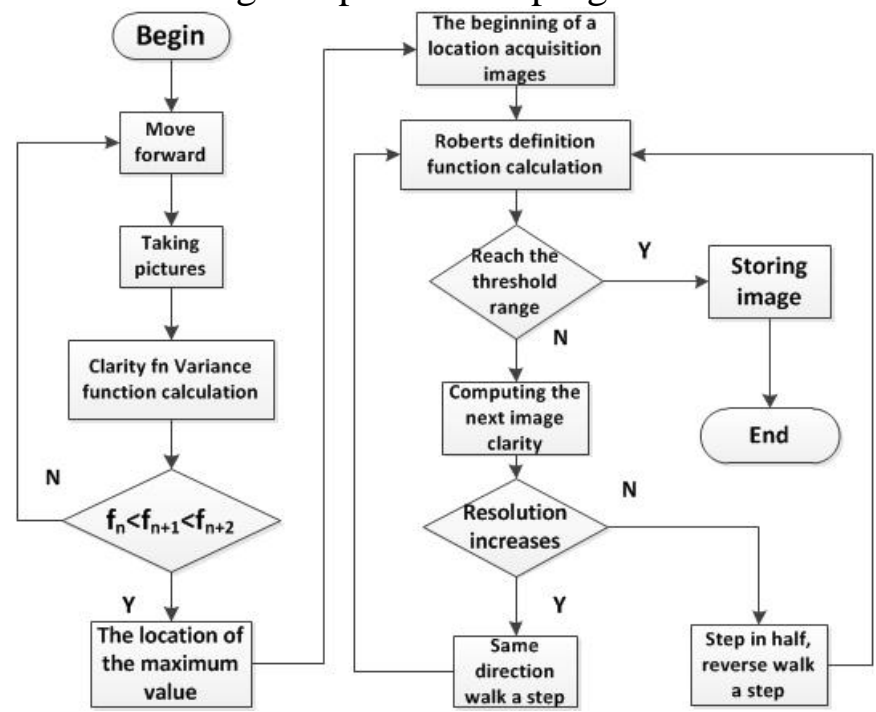

Fig.4 Flow chart of auto-focusing algorithm

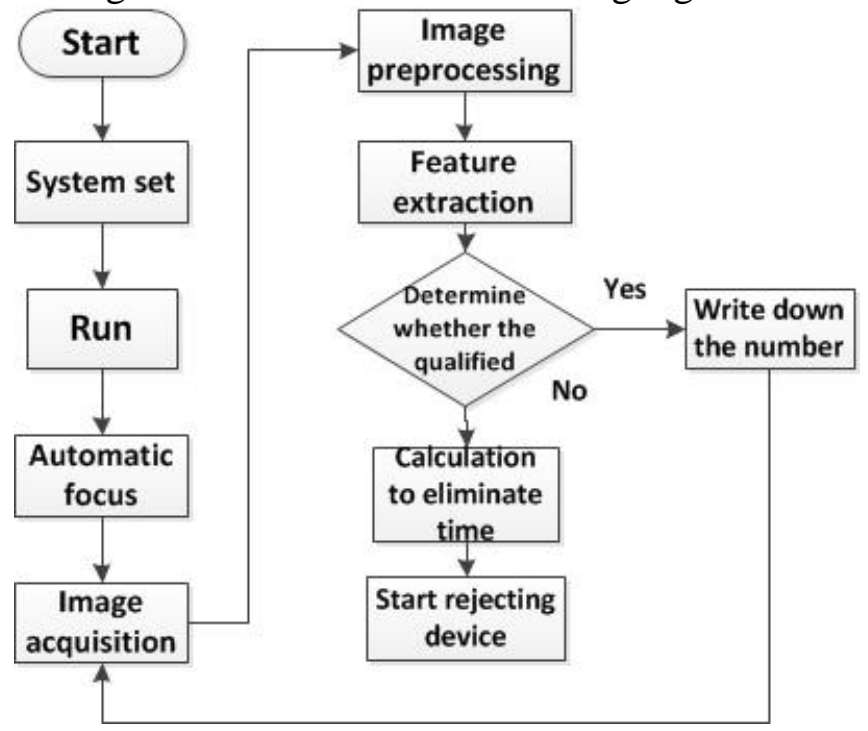

Fig.5 Overall software flow

The software of the industrial parts general testing platform based on machine vision includes parameter settings, image acquisition and image processing part. The system initialization includes the configuration of the STM32 USART, internal clock, interrupt and buttons. After the initialization, tested items are transferred into the detection area by the conveyor belt, and the Roberts gradient sum and Variance function are adopted to improve the initial focus. The lens goes to the appropriate position for image acquisition. The median filtering and binary segmentation[10] is used for image pre-processing. With the appropriate binary image segmentation, a clear image can be characterized. Then the target area is extracted to determine whether the part meets the setting conditions. If the condition is achieved, the accumulator automatically accumulate; if not, the pneumatic gripping hand holds the part to the specified folder defective area. The overall software flow is shown in Fig.5.

\section{The experiment and analysis}

In order to validate this system, two experiments are carried out. One is the image auto-focusing experiment, and the other is the machine running experiment. 

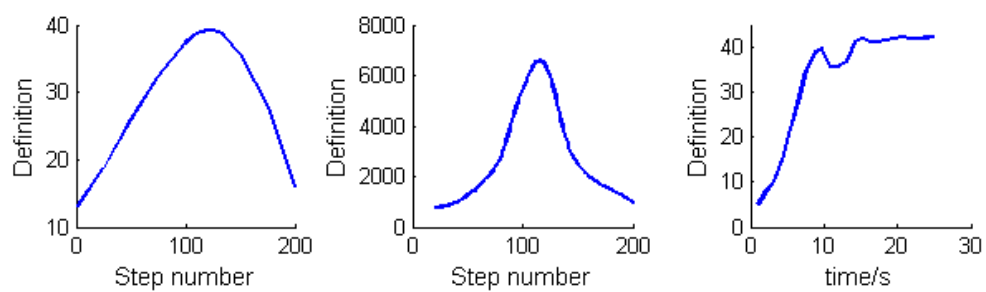

(a) Roberts gradient function

(b) Variance function (c) Roberts gradient function focusing
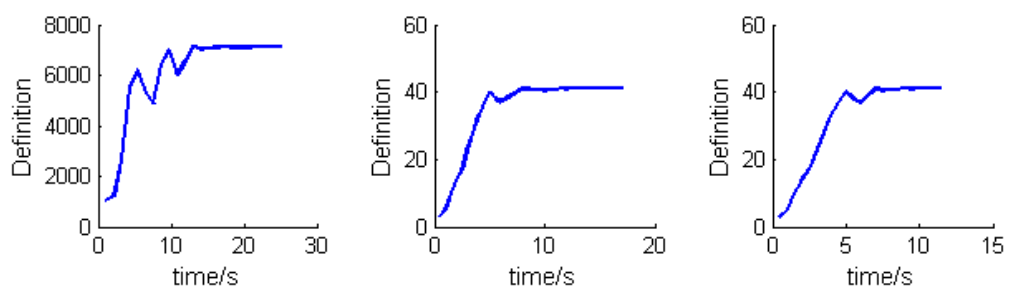

(d) Variance function of focusing

(e) Single step focus

(f) The overall focus

Fig.6Focusing curves for different algorithms

In the image auto-focusing experiment, the hybrid algorithm of the Roberts gradient sum function and Variance function is applied to the same image of the detected target. In this experiment, each step of the step motor is set to $0.0125 \mathrm{~mm}$. In Fig.6, the focusing results with different algorithms are present. The x coordinate is the focusing time with unit second. The $y$ coordinate is the definition of the image $\mathrm{f}(\mathrm{k})$. Fig.6(a) and (b) are the focusing results with single step running mode. It is seen from the curves that the Roberts gradient sum function and the Variance function both show smooth curves, only one extreme definition value, and almost the same running time. The sensitivity of the Variance function is greater than that of the Roberts gradient sum function, which is suitable for coarse tuning. The Roberts gradient and function has greater sensitivity near the focus, so it is suitable for fine-tuning. Fig.6(c) and (d) show the focusing results of both coarse and fine tune using the Roberts gradient sum function and Variance function, respectively. Fig.6(e) shows the results that the coarse adjustment adopts Variance function and fine tuning adopts the Roberts gradient single-step operation function. Fig.6(f) shows the results that coarse adjustment adopts Variance function and fine tuning adopts the Roberts gradient half step operation function. It can be seen from the curves that the Variance function greatly shortens the time of searching for peak area, and Roberts gradient sum function accurately find the adjustable focus in this area. On the whole, the combination of two kinds of function focusing algorithm greatly shortened the time focusing.

In the machine running experiment, first the system is initialized. The parts are placed on the conveyor belt for the first detection auto-focusing system after setting up the hardware and software parameters. When the lens position is fixed, and the same part is imaged 10 times, the time referred to as a single detection when the parts enter the check delivery platform to leave time to check delivery platform, as shown in table 1 . The average of the industrial components testing time is $3.246 \mathrm{~s}$, it can satisfy the requirement of real-time online detection. Fig.7(a), (b) and (c) are the focusing process images from the blur to clear process. Fig7(d) is a system software interface.

Table 1 Testing time

\begin{tabular}{lllllllllll}
\hline Number & 1 & 2 & 3 & 4 & 5 & 6 & 7 & 8 & 9 & 10 \\
\hline Time/s & 2.25 & 2.24 & 2.26 & 2.23 & 2.23 & 2.27 & 2.25 & 2.26 & 2.24 & 2.23 \\
Average/s & & & & & & 2.246 & & & & \\
\hline
\end{tabular}




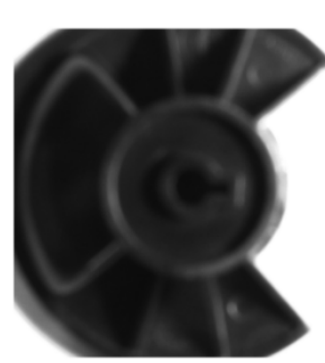

(a)

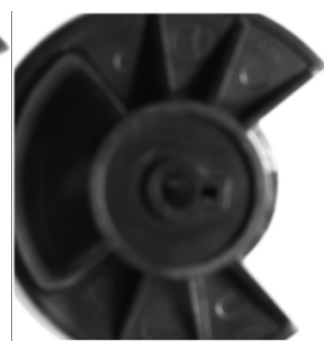

(b)

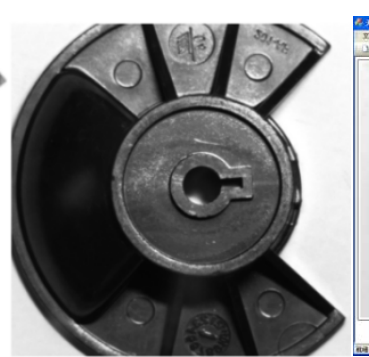

(c)

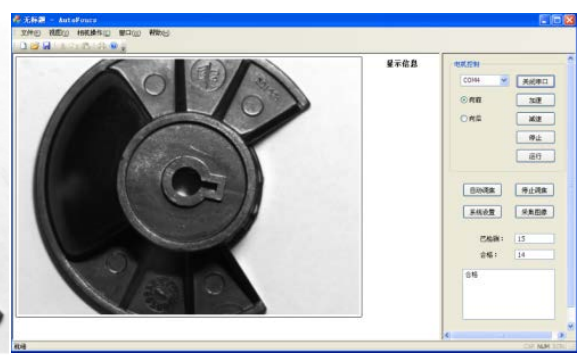

(d)

Fig.7 Experimental images and software interface

\section{Summary}

This paper presents the research on key technologies of the machine vision detection system. A high-speed detection system is developed, including the hardware configuration and software algorithms. The auto-focusing experiment and machine testing experiment are carried out to validate this detection system. In the image acquisition phase, the focusing algorithm combining variance function applying to coarse tuning and Roberts gradient sum function applying to fine tuning is proposed, which greatly reduces the time for collecting images. Experimental results show that the proposed optimization algorithm improves the detection efficiency, the machine detects running well. In the future, the following development will focus on the better performance of the system, and the application on real industrial products.

\section{Reference}

[1] Wu JiGang,Bin HongZan.Research and Development of Dimensional Inspection System for Thin Sheet Part Based on Machine Vision[J].Machine Tool And Hydraulics,2010, 17:86-88+101.

[2] Guo BaoYun.Research on High-Precision Methods of Visual Measurement of The Shape and Size of the Industrial Parts[J].Acta Geodaetica et Cartographica Sinica,2015,04:471.

[3] Liu JianQun,Kuang Hui,Ding ShaoHua.Research on Machine Vision System for Inspection of SMT Chip Pins[J].China Mechanical Engineering,2007,16:1908-1912.

[4] Wang Yu,Liu Min.Study of Machinery Rules Parts Detection Technology Based on Support Vector Machine[J].Electronic Measurement Technology,2012,01:80-84.

[5] Yang Yi,Zhong YiBo.External Clamping Device of Industry Manipulator Based on Tandem Cylinder-driven[J].Machine Tool And Hydraulics,2013,10:95-96.

[6] Li ShuangQiu,Yuan MingTing.Design and realization of power quality online monitoring devices based on STM32[J]. Modern Electronics Technique,2012,20:180-182+185.

[7] Dong XiaoQing,Huang JieXian,Zhang ShunYang.The key technology of stepping motor drive[J].Microcontrollers \& Embedded Systems,2008,06:14-17.

[8] Liu LiShuang,Sun ShuangHua,Lu NaiGuang.Study on The Selection Method of Focusing Function in Vision System[J].Journal of Beijing Institute of Machinery,2008,01:1-4.

[9] Ma HengKuo,Wei GuoQiang.Study on Stabilization of Auto Focusing Method Based on Image Processing[J].Aviation Precision Manufacturing Technology,2007,02:33-36.

[10] Luo Jun,Huang Jun.Two - Dimensional Precision Measurement System Based on Machine Vision[J].Computer Measurement \& Control,2007,01:11-13. 